\title{
Erratum: Yin et al. Remote Sensing of Atmospheric Hydrogen Fluoride (HF) over Hefei, China with Ground-Based High-Resolution Fourier Transform Infrared (FTIR) Spectrometry. Remote Sens. 2021, 13, 791
}

\author{
Hao Yin ${ }^{1,2} \mathbb{D}$, Youwen Sun ${ }^{1,3, *}$, Cheng Liu ${ }^{1,2,3,4,5}$, Wei Wang ${ }^{1}$, Changgong Shan ${ }^{1}$ and Lingling Zha ${ }^{6}$ \\ 1 Key Laboratory of Environmental Optics and Technology, Anhui Institute of Optics and Fine Mechanics, \\ Hefei Institutes of Physical Science, Chinese Academy of Sciences, Hefei 230031, China; \\ yhyh95@mail.ustc.edu.cn (H.Y.); chliu81@ustc.edu.cn (C.L.); wwang@aiofm.ac.cn (W.W.); \\ cgshan@aiofm.ac.cn (C.S.) \\ 2 Department of Precision Machinery and Precision Instrumentation, University of Science and Technology of \\ China, Hefei 230026, China \\ 3 Center for Excellence in Regional Atmospheric Environment, Institute of Urban Environment, Chinese \\ Academy of Sciences, Xiamen 361021, China \\ 4 Key Laboratory of Precision Scientific Instrumentation of Anhui Higher Education Institutes, University of \\ Science and Technology of China, Hefei 230026, China \\ 5 Anhui Province Key Laboratory of Polar Environment and Global Change, University of Science and \\ Technology of China, Hefei 230026, China \\ 6 School of Biology, Food and Environment, Hefei University, Hefei 230022, China; 1lzha@aiofm.ac.cn \\ * Correspondence: ywsun@aiofm.ac.cn
}

Citation: Yin, H.; Sun, Y.; Liu, C.; Wang, W.; Shan, C.; Zha, L.

Erratum: Yin et al. Remote Sensing of Atmospheric Hydrogen Fluoride (HF) over Hefei, China with Ground-Based High-Resolution Fourier Transform Infrared (FTIR) Spectrometry. Remote Sens. 2021, 13, 791. Remote Sens. 2021, 13, 1802. https://doi.org/10.3390/rs13091802

Received: 25 March 2021

Accepted: 3 April 2021

Published: 6 May 2021

Publisher's Note: MDPI stays neutral with regard to jurisdictional claims in published maps and institutional affiliations.

Copyright: (c) 2021 by the authors. Licensee MDPI, Basel, Switzerland. This article is an open access article distributed under the terms and conditions of the Creative Commons Attribution (CC BY) license (https:// creativecommons.org/licenses/by/ $4.0 /)$.
The authors would like to make the following correction of Authorship, Author Contributions and Acknowledgment.

We would like to remove the author Ziheng Song from the author list in [1] and move it into the thanks list because he is from high school and he just contributed validation, retrieval process, data curation and investigation. With the consent of all the authors, the author should be deleted. In addition, the content of "We thank Ziheng Song for his contribution to the work of validation, retrieval process, data curation and investigation" is added in the acknowledgment. We apologize for this mistake and state that the scientific conclusions are unaffected. The original article has been updated.

The correct version is as follows:

Hao Yin ${ }^{1,2}$, Youwen Sun ${ }^{1,3, *}$, Cheng Liu ${ }^{1,2,3,4,5}$, Wei Wang ${ }^{1}$, Changgong Shan ${ }^{1}$ and Lingling Zha ${ }^{6}$

${ }^{1}$ Key Laboratory of Environmental Optics and Technology, Anhui Institute of Optics and Fine Mechanics, Hefei Institutes of Physical Science, Chinese Academy of Sciences, Hefei 230031, China; yhyh95@mail.ustc.edu.cn (H.Y.); chliu81@ustc.edu.cn (C.L.); wwang@aiofm.ac.cn (W.W.); cgshan@aiofm.ac.cn (C.S.)

${ }^{2}$ Department of Precision Machinery and Precision Instrumentation, University of Science and Technology of China, Hefei 230026, China

${ }^{3}$ Center for Excellence in Regional Atmospheric Environment, Institute of Urban Environment, Chinese Academy of Sciences, Xiamen 361021, China

${ }^{4}$ Key Laboratory of Precision Scientific Instrumentation of Anhui Higher Education Institutes, University of Science and Technology of China, Hefei 230026, China

${ }^{5}$ Anhui Province Key Laboratory of Polar Environment and Global Change, University of Science and Technology of China, Hefei 230026, China

${ }^{6}$ School of Biology, Food and Environment, Hefei University, Hefei 230022, China; llzha@aiofm.ac.cn

*Correspondence: ywsun@aiofm.ac.cn 
Authors Contributions: Conceptualization, H.Y. and Y.S.; methodology, H.Y. and Y.S.; software, H.Y. and Y.S.; validation, H.Y.; Y.S.; W.W.; C.S. and L.Z.; formal analysis, H.Y. and Y.S.; investigation, H.Y.; Y.S.; W.W.; C.G. and L.Z.; retrieval process, H.Y.; Y.S. and L.Z.; data curation, H.Y.; Y.S.; W.W.; C.S. and L.Z.; writing-original draft preparation, H.Y. and Y.S.; writing - review and editing, H.Y. and Y.S.; visualization, H.Y.; supervision, C.L.; project administration, Y.S. and C.L.; funding acquisition, Y.S. and C.L. All authors have read and agreed to the published version of the manuscript.

Acknowledgments: The processing environment of SFIT4 and some plot programs are provided by the National Center for Atmospheric Research (NCAR), Boulder, CO, USA. The NDACC networks are acknowledged for supplying the SFIT software and advice. The TCCON networks are acknowledged for supplying the GFIT software and advice. The LINEFIT code is provided by Frank Hase, Karlsruhe Institute of Technology (KIT), Institute for Meteorology and Climate Research (IMK-ASF), Germany. We thank the FTIR groups at the University of Bremen, Germany, and the University of Wollongong, Australia, for their help in setting up the retrieval and operating the FTIR spectrometer at Hefei. We thank Ziheng Song for his contribution to the work of validation, retrieval process, data curation and investigation.

\section{Reference}

1. Yin, H.; Sun, Y.; Song, Z.; Liu, C.; Wang, W.; Shan, C.; Zha, L. Remote Sensing of Atmospheric Hydrogen Fluoride (HF) over Hefei, China with Ground-Based High-Resolution Fourier Transform Infrared (FTIR) Spectrometry. Remote Sens. $2021,13,791$. [CrossRef] 